\title{
BMJ Open Cost-utility analysis of transcranial direct current stimulation (tDCS) in non-treatment-resistant depression: the DISCO randomised controlled study protocol
}

Anne Sauvaget (10 , ${ }^{1,2}$ Lydie Lagalice, ${ }^{1}$ Solène Schirr-Bonnans, ${ }^{3}$ Christelle Volteau, ${ }^{4}$ Morgane Péré, ${ }^{4}$ Cécile Dert, ${ }^{3}$ Annabelle Rivalland, ${ }^{1}$ Fabienne Tessier, ${ }^{1}$ Adeline Lepage, ${ }^{1}$ Agathe Tostivint, ${ }^{1}$ Thibault Deschamps, ${ }^{2}$ Véronique Thomas-Ollivier, ${ }^{2}$ Alison Robin, ${ }^{2}$ Noémie Pineau, ${ }^{2}$ Clémence Cabelguen, Nicolas Bukowski, ${ }^{1}$ Marie Guitteny, ${ }^{1}$ Auxane Beslot, ${ }^{1}$ Luc Simons, ${ }^{1}$ HUGOPSY Network, ${ }^{5}$ Jean-Marie Vanelle, ${ }^{1}$ Giordano D'Urso, ${ }^{6}$ Samuel Bulteau, ${ }^{1,7}$ Valéry-Pierre Riche, ${ }^{3}$ DISCO investigators group

To cite: Sauvaget A, Lagalice L, Schirr-Bonnans S, et al. Costutility analysis of transcranial direct current stimulation (tDCS) in non-treatmentresistant depression: the DISCO randomised controlled study protocol. BMJ Open 2020;10:e033376. doi:10.1136/ bmjopen-2019-033376

- Prepublication history for this paper is available online. To view these files, please visit the journal online (http://dx.doi. org/10.1136/bmjopen-2019033376).

Received 01 August 2019 Revised 07 December 2019 Accepted 11 December 2019

Check for updates

(C) Author(s) (or their employer(s)) 2020. Re-use permitted under CC BY-NC. No commercial re-use. See rights and permissions. Published by BMJ.

For numbered affiliations see end of article.

Correspondence to

Pr Anne Sauvaget;

anne.sauvaget@chu-nantes.fr

\section{ABSTRACT}

Introduction Depression is among the most widespread psychiatric disorders in France. Psychiatric disorders are associated with considerable social costs, amounting to $€ 22.6$ billion for treatment and psychotropic medication in 2011. Treatment as usual (TAU), mainly consisting of pharmacotherapy and psychotherapy, is effective for only a third of patients and in most cases fails to prevent treatment resistance and chronicity. Transcranial direct current stimulation (tDCS) consists in a non-invasive and painless application of low-intensity electric current to the cerebral cortex through the scalp. Having proved effective in depressed patients, it could be used in combination with TAU to great advantage. The objective is to compare, for the first time ever, the cost-utility of tDCS-TAU and of TAU alone for the treatment of a depressive episode that has been refractory to one or two drug treatments.

Methods and analysis This paper, based on the DISCO study protocol, focuses on the design of a prospective, randomised, controlled, open-label multicentre economic study to be conducted in France. It will include 214 patients with unipolar or bipolar depression, assigning them to two parallel arms: group A (tDCS-TAU) and group B (TAU alone). The primary outcome is the incremental cost-effectiveness ratio, that is, the ratio of the difference in cost between each strategy to the difference in their effects. Their effects will be expressed as numbers of quality-adjusted lifeyears, determined through administration of the EuroQol Five-Dimension questionnaire over a 12-month period to patients (EQ-5D-5L). Expected benefits are the reduction of treatment resistance and suicidal ideation as well as social and professional costs of depression. Should depressionrelated costs fall significantly, tDCS might be considered an efficient treatment for depression.

Ethics and dissemination This protocol has been approved by a French ethics committee, the CPP--Est IV (Comité de Protection des Personnes-Strasbourg). Data are to be published in peer-reviewed medical journals.
Strengths and limitations of this study

The DISCO study protocol is the first economic evaluation comprising a comparative cost-utility analysis of transcranial direct current stimulation (tDCS) plus treatment as usual (TAU) versus TAU alone, for the treatment of depression.

- The study will yield new information to improve primary care of patients with non-resistant unipolar or bipolar depression.

- It has one of the longest follow-ups in brain stimulation research.

- The DISCO study is flexibly designed, calling for administration of tDCS at a frequency varying with the needs of individual patients - as opposed to applying a rigid timepoint protocol—which better reflects actual clinical practice and is of greater benefit to both patients and study centres.

- As a real-life study, the study does not involve changes in current patient treatments.

Trial registration number RCB 2018-A00474-51; NCT03758105

\section{INTRODUCTION}

\section{Background and rationale}

Major depressive disorder (MDD) is one of the most widespread psychiatric disorders worldwide. It has an estimated prevalence of $5 \%-12 \%$ in the French population ${ }^{12}$ and a substantial impact on patients' health and quality of life. A systematic analysis of the Global Burden Study in 2010 revealed that unipolar depression represents the second greatest cause of the increase of 
years lived with disability and that its prevalence had not declined over the preceding 20 years despite therapeutic advances. ${ }^{3}$ Psychiatric disorders also have a considerable socioeconomic cost: in 2011, treatments and consumption of psychotropic drugs in France accounted for $€ 22.6$ billion, or $16 \%$ of total health expenditures there. ${ }^{1}$ Despite appropriate treatments, $30 \%-40 \%$ of patients suffering from MDD show no improvement. ${ }^{4}$ Only a third of patients achieve clinical remission after one antidepressant drug treatment step, while up to four treatment steps are necessary to reach clinical remission for approximately $70 \%$ of patients. ${ }^{5}$ Moreover, an increased risk of relapse is reported for those who require more than one drug treatment to achieve remission. ${ }^{56}$ Treatment resistance is defined as the failure to achieve remission after at least two different antidepressant administration phases at an effective dosage over a period of 6 weeks. In these cases, higher dosages may be necessary, as well as combination and augmentation strategies. ${ }^{7-10}$ In $63 \%$ of patients, the failure of drug treatments can be explained by poor compliance, ${ }^{11}$ which in turn is often related to tolerability: approximately $85 \%$ of patients taking serotonin reuptake inhibitors present at least one adverse effect at the beginning of the treatment. ${ }^{12}$ Therefore, improving tolerability and acceptability of antidepressant treatments is paramount.

Transcranial direct current stimulation (tDCS) is a treatment technique consisting in the non-invasive and painless application of low-intensity electric current to the cerebral cortex through the scalp. No longer confined to the research setting, tDCS is now being used in everyday clinical practice and at new facilities entirely dedicated to psychiatric neuromodulation. ${ }^{13}$ It is a nonpharmacological psychiatric therapy that has proven effective in patients with $\mathrm{MDD}^{14}$ as well as those with other psychiatric and neurological conditions, including obsessive-compulsive disorder, ${ }^{15}$ schizophrenia, ${ }^{16}$ posttraumatic stress disorder, ${ }^{17}$ addiction, ${ }^{18}$ autism spectrum disorders ${ }^{19}$ and dementia. ${ }^{20}$

According to Canadian Network for Mood and Anxiety Treatments MDD management guidelines, tDCS's sister technique, repetitive transcranial magnetic stimulation (rTMS), has already reached the highest level of evidence of efficacy (level 1) in patients not responding after administration of one antidepressant. ${ }^{21}$ Compared with rTMS, tDCS is less studied but has the advantage of being easier to perform and less expensive. ${ }^{22}$

It has been demonstrated that patients with depressive disorder present hypofunction and cerebral abnormalities of the dorsolateral prefrontal cortex,${ }^{23}$ which can be reversed by means of anodal (excitatory) tDCS. ${ }^{24}$ Most meta-analyses show that active tDCS is significantly more effective than sham tDCS in terms of MDD patient response and remission rates. ${ }^{25-29}$ Although tDCS has also proved effective in treatment-resistant depression, ${ }^{30-33}$ the level of resistance is predictive of the degree of tDCS efficacy. It would thus appear that tDCS is ideally for use in patients with depressive disorder with a low level of resistance to pharmacological treatment. ${ }^{2534}$ To date, most studies evaluating tDCS treatment have focused on unipolar depression. ${ }^{35-37}$ Nevertheless, a recent meta-analysis including 13 studies showed a significant decrease of depression after tDCS treatment in bipolar depression. ${ }^{38}$ Interestingly, certain depressive symptoms were associated with a better response to tDCS treatment. ${ }^{39}$ Moreover, although tDCS represents a promising alternative treatment for depression but its popularity was lower than other neurostimulation therapies, its longterm efficacy has yet to be fully demonstrated. ${ }^{40}$ A study highlighted that $45 \%$ of patients were still in remission 3 months after 10 sessions of tDCS and that the relapse rate seemed to increase with the interval between tDCS sessions. ${ }^{41}$ Recent recommendations call for more studies with maintenance $\mathrm{tDCS}^{25}$ and long-term evaluation of the effect of tDCS in patients with depressive disorder. ${ }^{34}$ To date, no study has compared the long-term efficacy of TAU-tDCS and TAU-only treatments. We hypothesise that, in comparison with TAU alone, TAU-tDCS in patients with unipolar and bipolar depression at the early stages of an episode (one or two treatment failures) is cost-effective, preventing greater depression, optimising healthcare resource consumption and costs, improving patients' quality of life and allowing them to resume work sooner.

\section{Objectives and trial design}

The main purpose of this randomised controlled openlabel study is to perform a cost-utility analysis comparing tDCS-TAU (group A) and TAU-only (group B) treatments over 12 months in patients suffering from unipolar or bipolar depression after one or two antidepressant failures during the current episode, from a societal perspective. This will involve calculating the incremental cost-effectiveness ratio (ICER), which is the ratio of the difference in cost between each strategy to the difference in their effects, as expressed in quality-adjusted life-years (QALYs) measured by the EuroQol Five-Dimension questionnaire (EQ-5D-5L) (https://euroqol.org/).

Secondary outcomes to be evaluated are as follows:

For both groups at 12 months: response and remission rate; relapse rate and survival without relapse; progression of the depressive state; cognitive performance; drug tolerability; suicide attempt rate and suicide rate; changes in instructions concerning medication and declared medication compliance.

For both groups at 5 years: budget impact analysis from the point of view of the National Health Insurance (NHI) to evaluate the financial impact of tDCS through different scenarios including the diffusion percentage of the technology and its reimbursement.

For group A only, at 12 months, response rate at end of tDCS treatments; annual number of tDCS sessions needed to maintain response; duration of tDCS response after initial treatment and after following ones: tDCS compliance, tolerability and security; patient's acceptability of tDCS and how it is administered; identification 


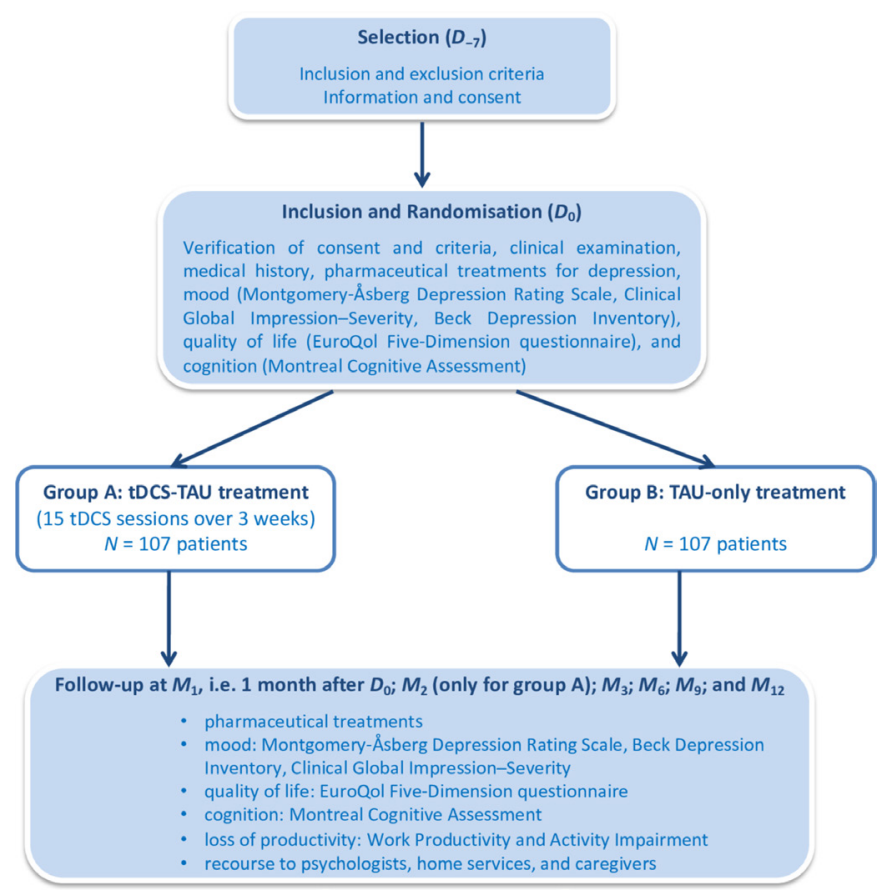

Figure 1 Design of DISCO protocol. $D_{n}$, day $n ; \mathrm{M}_{n}$, month $n$; tDCS, transcranial direct current stimulation; TAU, treatment as usual.

of response predictors and impact of tDCS implementation on healthcare organisation.

\section{METHODS AND ANALYSIS}

\section{Study setting and recruitment}

This is an ongoing, prospective, open-label, multicentre, randomised controlled study that shall recruit a total of 214 patients referred by either private or hospital psychiatrists previously informed about the study, randomly assigning half the patients to group A (experimental tDCS-TAU treatment) and half to group B (TAU-only treatment) for the purpose of comparison (figure 1). The study sponsor is Nantes CHU (Nantes University Hospital) in France. Patients will be included and treated at 12 centres in France. The study started in February 2019 and is planned to be completed in February 2022.

\section{Inclusion criteria}

All 214 patients must be over 18 years, present with a unipolar or bipolar depressive episode, as defined by the diagnostic criteria of the fifth edition of the Diagnostic and Statistical Manual of Mental Disorders, ${ }^{42}$ score $\geq 15$ on the Montgomery-Åsberg Depression Rating Scale (MADRS) and have failed to respond to one or two antidepressant drugs taken in succession or combination during the current episode. Each subject must be able to understand information given, make decisions, participate willingly, complete required questionnaires, take orally administered medication independently or with assistance throughout the study period and go to the study centre for follow-up visits. If patients agree to participate, they must give verbal consent to the medical investigator.

The current 'pragmatic' recruitment (ie, inclusion/ exclusion criteria) is sufficiently broad to include a large representative sample of patient suffering from depression eligible to $\mathrm{tDCS}$ treatment.

\section{Exclusion criteria}

Patients may not be enrolled in the study if they have received electroconvulsive therapy or rTMS during the current episode; are suffering a major depressive episode with mixed or psychotic symptoms; are schizophrenic; are addicted to any substance except nicotine; are epileptic; have undergone neurosurgery or have a significant neurodegenerative disease; suffer from a severe or progressive somatic disease; have a pacemaker, an intracerebral implant containing metal, or another device or condition contraindicating tDCS administration; are pregnant and nursing women or women of childbearing age who are not using contraception; are enrolled in another interventional clinical trial; are minors or persons whose freedoms are restricted due to a legal or administrative decision or who are hospitalised without their consent, under guardianship or are unable to agree with longitudinal follow-up.

\section{Study process}

During the screening visit, patient information and verbal consent are obtained, patients are checked against inclusion and exclusion criteria and a psychiatrist performs a clinical evaluation (table 1). Medical research teams are in charge of enrolment and assignment of each participant to group A or B.

\section{Inclusion visit and randomisation (day 0)}

During the baseline inclusion visit, 1 week before the first tDCS session, patients are again checked against inclusion and exclusion criteria. They are then assigned to two groups through permuted block randomisation using a computer program and their electronic case report forms while stratifying by centre. No other variable is taking into account for the randomisation.

\section{Follow-up}

Investigators will meet study participants from both groups 1, 6 and 12 months after randomisation $\left(M_{1}, M_{6}\right.$ and $M_{12}$ in table 1). Group A patients will have an additional visit at $M_{2}$, 4 weeks after the end of the initial 3-week round of tDCS sessions. Those group A patients who have responded to tDCS but whose condition is worsening at $M_{2}$ will be offered an additional tDCS round, while those who have not responded will revert to the TAU for their respective study centres. In addition to follow-up visits, patients will receive phone calls at $M_{3}$ and $M_{9}$.

The study will last 36 months, including 24 months for enrolment and 12 for treatment and longitudinal follow-up. Patients who drop out of the study will be offered TAU. Drop-out dates and reasons will be recorded. 


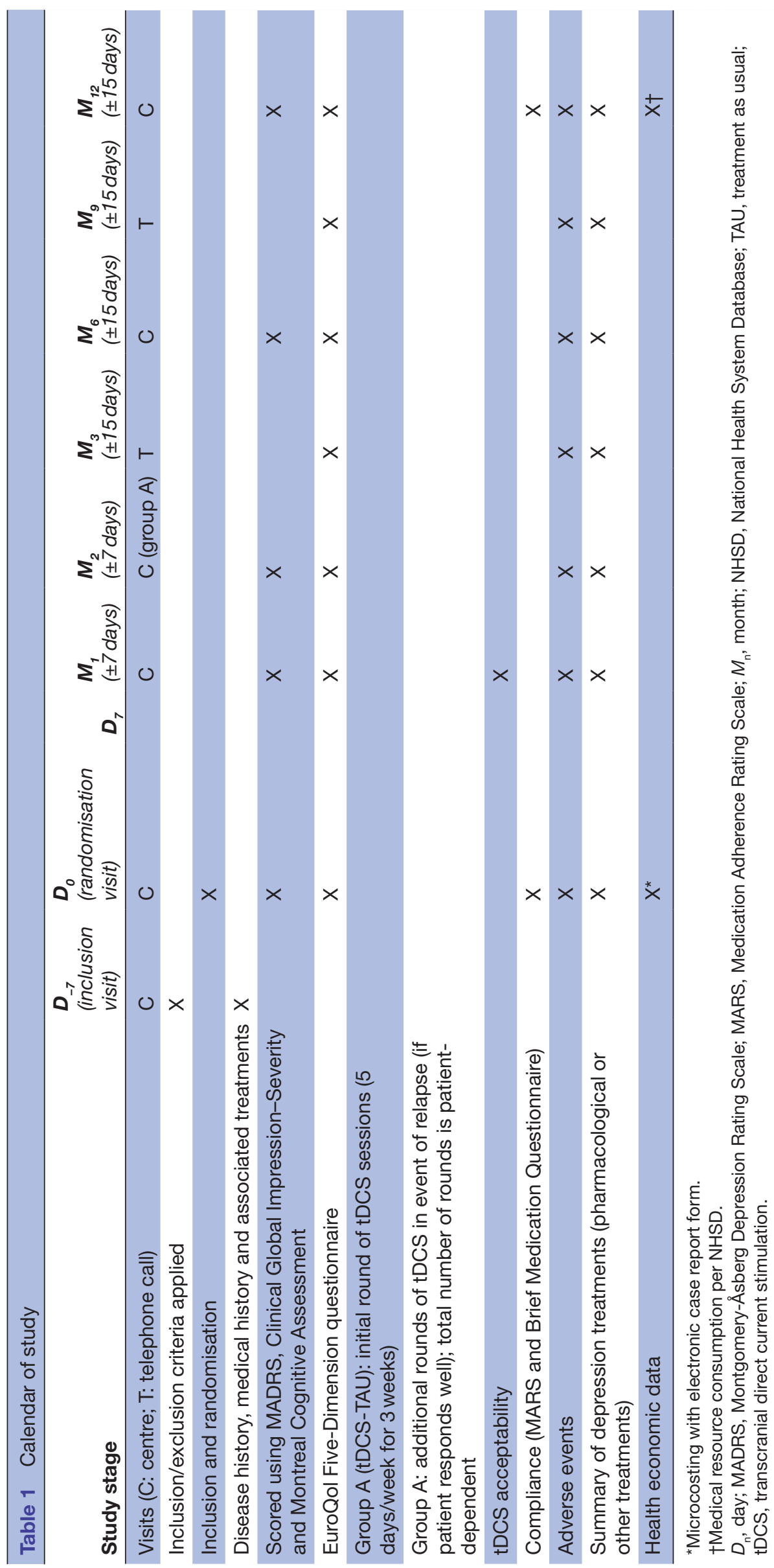

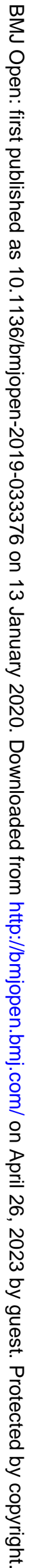




\section{Assessments}

The following information will be recorded during the baseline visit $\left(D_{0}\right)$ : sociodemographic data (age, gender, laterality, professional status and marital status), medical history (duration of illness, duration of the current episode and psychiatric, addictive or somatic comorbidities), and current treatment.

Quality of life will be assessed with the EQ-5D-5L questionnaire at $D_{0}, M_{1}, M_{6}, M_{12}$; during group A $M_{2}$ follow-up consultations and at $M_{3}$ and $M_{9}$ by phone. MADRS, Beck Depression Inventory and Clinical Global ImpressionSeverity scores will be collected at $D_{0}, M_{1}, M_{2}$ (group A only), $M_{6}$ and $M_{12}$. Additional MADRS scores will be collected at the start and end of any additional round of tDCS sessions for group A patients who have relapsed. The Montreal Cognitive Assessment will be administered at $D_{0}$, $M_{1}, M_{6}$ and $M_{12}$. Acceptability and tolerability of tDCS will be assessed by visual analogue scale and Comfort Rating Questionnaires, respectively, following the initial round of tDCS sessions (at $M_{1}$ ). Drug treatment compliance will be evaluated at $D_{0}$ and $M_{12}$ using the Medication Adherence Rating Scale, Compliance Rating Scale and Brief Medication Questionnaire. Compliance with the tDCS treatment plan will be evaluated by recording the actual number of tDCS sessions completed. At each visit, investigators will record any side effects and changes in treatment since the previous visit in the electronic case report form.

The cost-utility analysis will be performed with a societal perspective (ie, the broadest perspective), which means that costs to all stakeholders (NHI, hospitals, clinics, private insurances, patients) will be considered. Health services for all reasons will be included. Elements that may contribute to explain the differential in costs between the two groups are inpatient and outpatient health resources consumed, like outpatient psychiatric consultations, diagnosis-related groups, length of hospitalisations, pharmacotherapies, travel for medical care, medical imaging, laboratory analyses, nursing care, recourse to a psychologist or psychotherapist. Recourse to formal care (time spent by caregivers or home services) or informal care (time spent by relatives help), duration of work stoppage, loss of production capacity at the societal level and responses to Work Productivity and Activity Impairment questionnaires will also be collected. Data about the consumption of medical resources for which French NHI base prices exist will be extracted from the French National Health System Database (NHSD) (Système National des Données de Santé), which incorporates the French hospital expenditure database. Other resources consumed that are not covered by the NHI system will be collected via patient diaries fulfilled by the patient and reported into an electronic case report form. A microcosting approach will be used to evaluate the production cost of a tDCS session for the hospital; it will cover 15 'first sessions' and the 15 'following sessions' of tCDS in three centres participating in the study. The microcosting will consider time spent by staff, resources used including tDCS medical device.
When patients come in for follow-up visits, a survey on organisational impact (secondary end point) will be administered to each participating hospital team. The organisational impact will be assessed according to criteria extracted from the evaluation grid developed by Roussel et $a l^{43}$

We will carry out a 5-year budget impact analysis from the point of view of the NHI to evaluate the financial impact of tDCS through different scenarios, including the diffusion percentage of the technology and its reimbursement. To do this, we will assume a closed cohort over a year and use the 1 year management cost obtained in the cost-utility analysis.

\section{Interventions}

Two kinds of tDCS stimulator will be used in this study: the Soterix (Soterix Medical, New York, USA) and Sooma (Sooma Oy, Helsinki, Finland) devices, both of which bear $\mathrm{CE}$ product conformity marks. Units will be equally distributed between the 12 study centres. The montage allows for anodal stimulation of the left dorsolateral prefrontal cortex and cathodal stimulation of the right orbitofrontal cortex. ${ }^{24}$ On $D_{7}$, 1 week after randomisation, group A patients will commence an initial round of 30 min 2-mA tDCS treatment sessions every weekday for 3 weeks (15 sessions total). If they respond to tDCS but have relapsed, they will be offered an additional round of tDCS sessions following the $M_{2}$ assessment. If they are not responsive to tDCS, their respective centres are free to apply any therapeutic strategy.

TAU is defined as a wide range of standard and available treatments for major depressive and bipolar disorders, such as psychotherapy, medications and non-invasive brain stimulations.

\section{Outcomes}

The primary end point in our study is the ICER, comparing tDCS-TAU and TAU-only treatments for unipolar and bipolar depression, from a societal perspective, at $M_{12}$ :

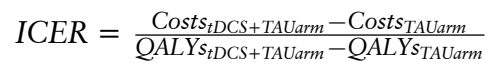

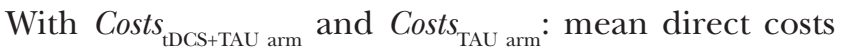
per patient in each arm.

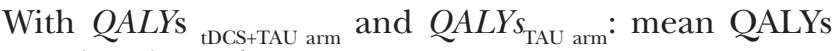
per patient in each arm.

QALYs will be determined using EQ-5D-5L questionnaires administered at seven time points. QALYs will be calculated using area under the curve methodology ${ }^{44}$ and the weighting coefficients that will be provided soon in France for the EQ-5D-5L. If they are not available at the time of analysis, a mapping between EQ-5D-3L and EQ-5D-5L will be achieved in order to get relevant weights. The cost-utility analysis will be performed in accordance with French Health Authority (HAS) recommendations. ${ }^{45}$

Direct costs will be included in the primary analysis taking into account all stakeholders (NHI, hospitals, private insurance companies, patients and caregivers) to acquire the desired societal perspective. In a secondary 
analysis, direct and indirect costs will be included. While direct costs relate to the resources needed for the production of the interventions being studied, other resources may be made unavailable because of the mortality and/ or morbidity. These lost resources, as productivity loss, are included as indirect costs. The 12-month period following randomisation affords time to estimate the impact of tDCS on patient quality of life and costs during depressive episodes, detect relapses or recurrences and identify potential medication complications. No cost or QALY discount will be applied given the duration of this period.

Cost estimation will follow three steps: (1) identification and quantification of resources consumed, (2) determination of unit costs for resources and (3) multiplication of resource quantities by corresponding unit costs to estimate total costs. Resources used will be determined using the French NHSD and recorded on participants' electronic case report forms. Direct costs will be determined by applying NHI base prices. Costs of psychologist appointments and home services-as these are assigned no NHI base prices-will be determined using the national mean fee and national mean cost, respectively. Indirect costs, as reflected by loss of production during work stoppage and responses to Work Productivity and Activity Impairment questionnaires, will be evaluated by applying a national mean wage to work stoppage durations (human capital approach). Informal care will be valued by applying the national mean cost for domestic helpers to the time spent by caregivers.

\section{Statistical methods}

\section{Sample size calculation}

To our knowledge, no published study has evaluated the quality of life, healthcare resource consumption and costs associated with tDCS treatment for depression, meaning there is no precedent for establishing the number of subjects needed for our study with the formula by Glick. ${ }^{46}$ We therefore determined this number using a clinical efficacy criterion.

We hypothesised tDCS-TAU response rate of $60 \%$ at $M_{12}{ }^{14} 47$ and a TAU-only response rate of $40 \% .^{5}$ Assuming a 5\% (two-tailed) type I error, a power of $80 \%$ and an attrition rate of $10 \%$, a total of 214 subjects are required (107 subjects per arm) according to SAS software V.9.4.

\section{Principal end point: cost-utility analysis}

The principle of 'intention to treat' will be applied when performing the cost-utility analysis. Where necessary, missing data will be imputed. Data extracted from the French NHSD are considered as complete and do not necessitate imputation, but missing data from QALYs and costs (only for the non-reimbursed health resources) will be imputed with multiple imputations by chained equations (MICE), according to the method proposed by Faria $e t a l .{ }^{48}$ Analyses with and without imputed data will be presented. The primary analysis will be the analysis with imputed data.
Costs per patient will be presented in each arm in a table, disaggregated in cost items (eg, hospitalisations, medical visits, drugs, etc) with their mean and $95 \%$ CI. The total mean costs, total mean QALYs, mean life years gained per patient in each arm and the differences in costs and in QALYS between groups will be presented in another table with their mean and 95\% CI. The ICER will be presented in this latter table. The 95\% CIs will be calculated (two possible approaches to do so: by non-parametric bootstrapping ${ }^{49}$ or seemingly unrelated regression (SUR) $).^{48}$ If the baseline quality of life differs between groups $\mathrm{A}$ and $\mathrm{B}$, adjustment will be performed (two possible approaches to do so: either in the SUR, or in the regression that is performed before bootstrapping). Results will be tabulated.

For the decision criteria of cost-effectiveness, we arbitrarily chose a value of $€ 50000 /$ QALY because a threshold value for a QALY has yet to be recommended in France. This value seems to be acceptable in France. Results will also be presented using an acceptability curve showing the probability that $\mathrm{tDCS}-\mathrm{TAU}$ is cost-effective compared with TAU-only treatment using several values of societal willingness to pay for a QALY. A sensitivity analysis will be performed to assess the robustness of the results, through variation of some relevant parameters (eg, national mean fees, national mean costs, respectively for psychologist visits or home services).

Clinical end points: quality of data collected, population analysed and statistical methods

Data will be reviewed at the end of the study, prior to statistical analysis. The aim will be to identify the progress made and potential problems, and to classify any minor or major deviations.

The primary analysis population will be the 'intentionto-treat population', consisting of all the randomised patients. In addition, a sensitivity analysis will be conducted on the 'per-protocol' population, which includes patients who most closely adhere to the protocol (compliance with inclusion and non-inclusion criteria, lack of major protocol deviations and availability of primary end point data).

Descriptive analysis of the data collected during each patient's evaluation will be performed up until the final evaluation. Both subject groups will be analysed at $D_{0}$ (baseline) and $M_{12}$. Continuous variables will be described using quartiles, means, SD and extreme values, while frequencies and percentages will be used for qualitative variables.

Remission, response and relapse rates (and their corresponding $95 \%$ CIs) at $M_{12}$ for groups A and B will be compared using the generalised linear model (logistic model) and considering study centre to be a random factor (randomisation stratification factor). Relapsefree survival rates until $M_{12}$ will be estimated using the Kaplan-Meier method and compared between two groups through the log-rank test using stratification by study centre. Sustainability (or maintenance) of the therapeutic 
response over time will be assessed through Cox multivariate analysis.

Changes in MADRS, Clinical Global ImpressionSeverity and Montreal Cognitive Assessment scores for both groups between $D_{0}$ and $M_{12}$ will be compared using linear mixed regression models and making study centre a random factor. The model will be adjusted according to $D_{0}$ scores. $M_{2}$ scores, only available for group A participants, will not be included.

Pharmacological treatment modifications and side effects will be described and compared by participant group using a $\chi^{2}$ test (or Fisher's exact test, if appropriate). The numbers and percentages of suicidal attempts for each group will be compared using Fisher's exact test.

Medication Adherence Rating Scale, Compliance Rating Scale and Brief Medication Questionnaire scores for each group at $M_{12}$ will be compared using linear mixed regression models and making study centre a random factor. Non-compliance percentages will also be determined for both groups.

For all outcomes, mean differences between the two groups, and the corresponding $95 \%$ CIs, will be reported. The following criteria will be described at $D_{0}$ and $M_{12}$ for group A patients: tDCS response rate; number of tDCS rounds prescribed; efficacy and side effects of each tDCS round; compliance rate and the acceptability of the procedure, measured using the visual analogue scale.

Missing data will be described for both groups. If required, imputation will be performed for missing data on clinical response, remission and relapse. First, it will be assumed that the patients concerned exhibited no response, remission or relapse. Second, sensitivity analyses will be performed using MICE on the basis of main patient characteristics. For clinical criteria other than response, remission and relapse, there will be no imputation. Secondary clinical outcomes (MADRS, Clinical Global Impression-Severity and Montreal Cognitive Assessment scores) will not be imputed because they are analysed using a repeated measures mixed model that is robust to missing data.

Analyses will be performed with SAS statistical software (SAS Institute). Statistical tests will be two-sided, and $\mathrm{p}$ values $<0.05$ shall be deemed statistically significant.

\section{Patient and public involvement}

There was neither patient nor public involvement in the development of this study protocol.

\section{ETHICS AND DISSEMINATION Consent}

Information about the study's aim and procedures is provided to all volunteer participants both verbally and in writing. Patients must provide oral consent to participate in the study. They are informed that participation is voluntary and that they may withdraw their consent at any time.

\section{Dissemination policy}

Data from the study will be published in a peer-reviewed medical journal.

\section{DISCUSSION}

The current challenges faced in the treatment of depressive disorders are to reduce relapses, recurrences and chronicity, and to optimise the consumption of healthcare resources for the management of depression. To meet these challenges, the prevention of pharmacological resistance and the promotion of adherence through better treatment tolerability, acceptability, feasibility and accessibility to patients as a first resort are paramount. Non-invasive brain stimulation, through techniques such as rTMS and tDCS, are recognised as effective therapeutic alternatives for the treatment of depressive disorders, boasting high levels of adherence and tolerability in comparison with pharmacological treatments. The rTMS procedure has proved more effective than antidepressants after a first treatment failure for MDD in newly diagnosed patients or at least two well-conducted antidepressant treatments. ${ }^{50}$ To date, no cost-effectiveness study has been conducted for tDCS in depression, although there have been cost-effectiveness studies that compared rTMS with medication ${ }^{5051}$ and with electroconvulsive therapy. ${ }^{52} 53$ The tDCS procedure has the advantage of being easier to perform and less expensive than rTMS. ${ }^{22}$ In two previous studies assessing the production costs of rTMS and tDCS (respectively) for the treatment of depression, we found tDCS to be less expensive. ${ }^{545}$ The DISCO study protocol is the first economic evaluation to use cost-utility analysis for the comparison of tDCS-TAU and TAU-only treatments in patients with depression. The French government has yet to determine a base price for tDCS procedures. In a preliminary paper, we estimated that the hospital production cost of a tDCS treatment for depression is $€ 1555.60$ per patient. ${ }^{55}$ DISCO will use microcosting to more accurately estimate this production cost.

This study will compare the costs of tDCS-TAU and TAU-only treatments by calculating volumes of resources consumed, determining the unit costs of these resources and multiplying the calculated volumes by the unit costs. To evaluate the overall cost of depression, estimation of direct medical and technical costs will not suffice because the indirect costs are not trivial and cannot be ignored. Thus, DISCO will address direct costs through its primary analysis and indirect costs through a secondary analysis, in accordance with HAS recommendations. ${ }^{45}$ To estimate indirect costs, the study will consider loss of production due to work stoppage-applying the human capital approach-and responses to Work Productivity and Activity Impairment questionnaires data on the involvement of caregivers will also be collected through electronic case report form questionnaires, to measure the informal costs of depression. 
From a clinical perspective, DISCO will offer new insights concerning primary care of patients with nontreatment-resistant unipolar or bipolar depression. ${ }^{25} 34$ It also boasts one of the longest follow-ups among brain stimulation studies. ${ }^{56}$

As any cost-effectiveness study protocols, DISCO strives to reflect clinical realities. The long-term effects of tDCS in depression are still poorly understood. In one study, 3 months after a 10 -session tDCS treatment programme, $45 \%$ of the patients were still in remission. ${ }^{41}$ It appears that depression relapse rates are higher when protocols call for entailing weekly session at a bi-monthly rate, ${ }^{5758}$ and when treatment resistance are higher at baseline. ${ }^{58}$ More recent publications therefore recommend studies with longer follow-ups ${ }^{34}$ and maintenance tDCS ${ }^{25}$ One of the secondary aims of DISCO is to determine the number of tDCS sessions needed to maintain remission in patients with depressive disorder responding to an initial round of tDCS sessions in combination with TAU. Further tDCS sessions will only be offered if patients respond to tDCS and then relapse. This flexible design, tailored to participants' unique needs, more closely models real clinical practice than a rigid timepoint protocol, and it benefits both patients and study centres.

As a real-life study, DISCO does not call for changes in current treatments. Furthermore, adjuvant tDCS has shown the potential to synergistically increase the efficacy of both pharmaceutical (including antidepressant) and non-pharmaceutical treatments. ${ }^{475}$

In conclusion, we assume that the addition of $\mathrm{tDCS}$ will offer greater efficacy than the usual treatments aloneas reflected by quality of life, number of hospitalisations, drug consumption, and societal repercussions. If our main hypothesis is confirmed, this study will provide evidence in support of tDCS as an add-on treatment for patients with non-treatment-resistant depression, and it will demonstrate-for the first time ever-that it is an efficient and effective therapeutic strategy. This would in turn offer justification for government reimbursement of tDCS procedures in France-and perhaps in other countries.

\section{Trial status}

Patient recruitment is in progress.

\section{Author affiliations}

${ }^{1} \mathrm{CHU}$ de Nantes, Addictology and Liaison Psychiatry Department, Hospital University of Nantes, Nantes, France

${ }^{2}$ Nantes Université, CHU Nantes,Movement, Interactions, Performance (MIP), EA 4334, University of Nantes, Nantes, France

${ }^{3} \mathrm{CHU}$ de Nantes, Innovation Cell, Partnership and Innovation Department,

Directorate of Medical Affairs and Research, University Hospital Centre Nantes, Nantes, France

${ }^{4} \mathrm{CHU}$ de Nantes, Section of Methodology and Biostatistics, University Hospital

Centre Nantes, Nantes, Pays de la Loire, France

${ }^{5}$ Réseau HUGOPSY, Université de Rennes, Rennes, France

${ }^{6}$ Department of Neurosciences, Reproductive Sciences, and Odontostomatology,

University of Naples Federico II, Napoli, Campania, Italy

${ }^{7}$ Inserm, SPHERE U1246, University of Nantes, Nantes, Pays de la Loire, France
Twitter Fabienne Tessier @Fabienne TESSIER

Acknowledgements The authors would like to thank Mr Jason Miller for the editing of the manuscript.

Collaborators DISCO investigators group: Bénédicte Gohier (Department of Psychiatry and Addictology, Angers University Hospital, University, UPRES EA 4638, University of Angers, 49100 Angers), Marine Rozet (Department of Psychiatry and Addictology, Angers University Hospital, University, UPRES EA 4638, University of Angers, 49100 Angers), Djamila Bennabi (Service de Psychiatrie, CIC-1431 INSERM, $\mathrm{CHU}$ de Besançon, F-25000 Besançon, France ; laboratoire de Neurosciences, Université Bourgogne Franche-Comté, F-25000 Besançon, France), Emmanuel Haffen (Service de Psychiatrie, CIC-1431 INSERM, CHU de Besançon, F-25000 Besançon, France ; laboratoire de Neurosciences, Université Bourgogne FrancheComté, F-25000 Besançon, France), Christophe Daudet (Clinique Mirambeau 22 Avenue De Maignon, 64600 Anglet, France), Ludovic Samalin (CHU ClermontFerrand, Department of Psychiatry, University of Clermont Auvergne, EA 7280, Clermont-Ferrand, France; Fondation Fondamental, Créteil, France), Pierre-Michel Llorca (CHU Clermont-Ferrand, Department of Psychiatry, University of Clermont Auvergne, EA 7280, Clermont-Ferrand, France; Fondation Fondamental, Créteil, France), Benoit Trojak (University Hospital of Dijon, Department of Psychiatry and Addictology, 21079 Dijon, France), Jean-Christophe Chauvet-Gélinier (Psychiatry unit, department of neurosciences, university hospital, $\mathrm{CHU}$ de Le Bocage, Bâtiment Marion, 14, rue Gaffarel, 21000 Dijon, France; Inserm LNC-UMR 1231, 21000 Dijon, France), Emmanuel Poulet (INSERM, U1028; CNRS, UMR5292; Lyon Neuroscience Research Center, Psychiatric Disorders: from Resistance to Response Team, Lyon, F-69000, France; University Lyon 1, Villeurbanne, F-69000, France; Department of Emergency Psychiatry, University Hospital Edouard Herriot, Hospices civils de Lyon, Lyon, France), Charline Magnin (INSERM, U1028; CNRS, UMR5292; Lyon Neuroscience Research Center, Psychiatric Disorders: from Resistance to Response Team, Lyon, F-69000, France; University Lyon 1, Villeurbanne, F-69000, France; Department of Emergency Psychiatry, University Hospital Edouard Herriot, Hospices civils de Lyon, Lyon, France), Stéphane Mouchabac (Sorbonne Universités, département de psychiatrie adulte et de psychologie médicale, AP-HP, hôpital Saint-Antoine, Paris, France), Ghina Harika Germaneau (Université de Poitiers, Unité de recherche clinique intersectorielle en psychiatrie à vocation régionale Pierre Deniker du Centre Hospitalier Henri Laborit F-86022 France ; Groupement De Recherche CNRS 3557), Nemat Jaafari (Université de Poitiers, Unité de recherche clinique intersectorielle en psychiatrie à vocation régionale Pierre Deniker du Centre Hospitalier Henri Laborit F-86022 France; Groupement De Recherche CNRS 3557), Dominique Drapier (Centre Hospitalier Guillaume Régnier, 108 avenue général leclerc 35703 Rennes ; EA 4712 Comportements et noyaux gris centraux, Université Rennes 1, France), Jean-Marie Batail (Centre Hospitalier Guillaume Régnier, 108 avenue général leclerc 35703 Rennes ; EA 4712 Comportements et noyaux gris centraux, Université Rennes 1, France), Maud Rotharmel (Service Hospitalo-Universitaire, Centre Hospitalier du Rouvray, 4, rue Paul Eluard, 76300 Sotteville-lès-Rouen, France), Caroline Berjamin (Service Hospitalo-Universitaire, Centre Hospitalier du Rouvray, 4, rue Paul Eluard, 76300 Sotteville-lès-Rouen, France), El-Hage Wissam (CHRU de Tours, Pôle de Psychiatrie, Tours, France ; UMR 1253, iBrain, Université de Tours, Inserm, Tours, France ; CIC 1415, Centre d'Investigation Clinique, Inserm, CHRU de Tours, Tours, France), Desmidt Thomas (CHRU de Tours, Pôle de Psychiatrie, Tours, France ; UMR 1253, iBrain, Université de Tours, Inserm, Tours, France).

Contributors AS, CV, SS-B, MP, AT, SB, J-MV, V-PR and MG designed the study and developed its protocol. AS is the study coordinator. AS, LL, GD'U, V-PR and SCB wrote the first version of the manuscript. AS, SB, CC, NB, MG, AB, LS, the HUGOPSY network, the DISCO investigators group are investigators and responsible for enrolment, determining treatment indications and data collection. All the authors (AS, LL, SS-B, CV, MP, CD, AR, FT, AL, AT, TD, VT-O, AR, NP, CC, NB, MG, AB, LS, BG, MR, DB, EH, CD, LS, P-ML, BT, J-CC-G, EP, CM, SM, NJ, GHG, DD, J-MB, MR, CB, $\mathrm{E}-\mathrm{HW}, \mathrm{TD}, \mathrm{J}-\mathrm{MV}, \mathrm{GD} \mathrm{U}, \mathrm{V}-\mathrm{PR}$ ) are involved in the data acquisition. $\mathrm{CD}$ is the project manager, helped with general organisation and sought ethical and regulatory approval. FT and AR are projects manager assistants. FT, AR, AP, AL, AR and LL contributed to tDCS administration and treatment planning. V-PR, MP and CV are responsible for calculating statistical power and performing statistical analyses. FT, $\mathrm{LL}, \mathrm{TD}, \mathrm{VT}-\mathrm{O}, \mathrm{NP}$ and $\mathrm{AB}$ are in charge of planning, randomisation and completion of electronic case report forms. All the authors read the draft critically, and approved the final version. All the authors agreed to be accountable for all aspects of the work.

Funding This work was supported by the French Ministry of Health (project PRME-17-0097).

Competing interests None declared. 
Patient consent for publication Not required.

Ethics approval This study has been approved by a French ethics committee, the CPP-Est IV (Comité de Protection des Personnes-Strasbourg) (RCB no. 2018A00474-51; ERB no. 18/75) and designed in accordance with the Declaration of Helsinki (final 2004 version) and French legislation (articles L1121-160 and L1126-7 of the French public health code).

Provenance and peer review Not commissioned; externally peer reviewed.

Open access This is an open access article distributed in accordance with the Creative Commons Attribution Non Commercial (CC BY-NC 4.0) license, which permits others to distribute, remix, adapt, build upon this work non-commercially, and license their derivative works on different terms, provided the original work is properly cited, appropriate credit is given, any changes made indicated, and the use is non-commercial. See: http://creativecommons.org/licenses/by-nc/4.0/.

\section{ORCID iD}

Anne Sauvaget http://orcid.org/0000-0002-1822-8535

\section{REFERENCES}

1 CNAMTS. Améliorer la qualité du système de santé et maîtriser les dépenses: propositions de l'Assurance maladie pour 2014. Rapport au ministre chargé de la Sécurité sociale et au Parlement sur l'évolution des charges et des produits de l'Assurance maladie au titre de 2014. [Improving the quality of the healthcare system and controlling expenses: Proposals from the French Health Insurance Administration for 2014. Report to the Minister of Social Security and to the Parliament on changes to products and costs for the French Health Insurance Administration in 2014. Paris: CNAMTS, 2013.

2 Fond G, Lancon C, Auquier P, et al. Prevalence of major depression in France in the general population and in specific populations from 2000 to 2018: a systematic review of the literature. Presse Med 2019;48:365-75.

3 Vos T, Flaxman AD, Naghavi M, et al. Years lived with disability (YLDs) for 1160 sequelae of 289 diseases and injuries 1990-2010: a systematic analysis for the global burden of disease study 2010 . Lancet 2012;380:2163-96.

4 Haute Autorité de Santé. Prise en charge des complications évolutives d'un épisode dépressif caractérisé de l'adulte. Recommendations. Avril 2007. [Treatment of long-term complications of major depressive disorder. Recommendations. April 2007.]. Paris: Haute Autorité de Santé, 2007.

5 Rush AJ, Trivedi MH, Wisniewski SR, et al. Acute and longerterm outcomes in depressed outpatients requiring one or several treatment steps: a STAR ${ }^{\star}$ D report. AJP 2006;163:1905-17.

6 Fekadu A, Wooderson SC, Markopoulo K, et al. What happens to patients with treatment-resistant depression? A systematic review of medium to long term outcome studies. J Affect Disord 2009;116:4-11.

7 Sachs GS, Thase ME, Otto MW, et al. Rationale, design, and methods of the systematic treatment enhancement program for bipolar disorder (STEP-BD). Biol Psychiatry 2003;53:1028-42.

8 Charpeaud C, Moliere F, Bubrovszky M, et al. Dépression résistante: les stratégies de changement et d'association de médicaments antidépresseurs. [Treatment-resistant depression: strategies for switching or combination antidepressants.]. Presse Med 2016;45:329-37.

9 Doumy O, Bennabi D, El-Hage W, et al. Dépression résistante: les stratégies de potentialisation. [Treatment-resistant depression: potentiation strategies.]. Presse Med 2016;45:338-49.

10 Saba G, Nieto I, Bation R, et al. Other therapeutic strategies. Presse Med 2016;45:323-32.

11 Pampallona S, Bollini P, Tibaldi G, et al. Patient adherence in the treatment of depression. Br J Psychiatry 2002;180:104-9.

$12 \mathrm{XH} \mathrm{H}$, Bull SA, Hunkeler EM, et al. Incidence and duration of side effects and those rated as bothersome with selective serotonin reuptake inhibitor treatment for depression: patient report versus physician estimate. J Clin Psychiatry 2004;65:959-65.

13 Sauvaget A, Poulet E, Mantovani A, et al. The psychiatric neuromodulation unit: implementation and management. J Ect 2018;34:211-9.

14 Brunoni AR, Tortella G, Benseñor IM, et al. Cognitive effects of transcranial direct current stimulation in depression: results from the SELECT-TDCS trial and insights for further clinical trials. J Affect Disord 2016;202:46-52.

15 D'Urso G, Brunoni AR, Mazzaferro MP, et al. Transcranial direct current stimulation for obsessive-compulsive disorder: a randomized, controlled, partial crossover trial. Depress Anxiety 2016;33:1132-40.
16 Mondino M, Haesebaert F, Poulet E, et al. Fronto-Temporal transcranial direct current stimulation (tDCS) reduces sourcemonitoring deficits and auditory hallucinations in patients with schizophrenia. Schizophr Res 2015;161:515-6.

17 D'Urso G, Mantovani A, Patti S, et al. Transcranial direct current stimulation in obsessive-compulsive disorder, posttraumatic stress disorder, and anxiety disorders. J. Ect 2018;34:172-81.

18 Sauvaget A, Trojak B, Bulteau S, et al. Transcranial direct current stimulation (tDCS) in behavioral and food addiction: a systematic review of efficacy, technical, and methodological issues. Front Neurosci 2015;9.

19 D'Urso G, Bruzzese D, Ferrucci R, et al. Transcranial direct current stimulation for hyperactivity and noncompliance in autistic disorder World J Biol Psychiatry 2015;16:361-6.

20 Ferrucci R, Mrakic-Sposta S, Gardini S, et al. Behavioral and neurophysiological effects of transcranial direct current stimulation (tDCS) in fronto-temporal dementia. Front Behav Neurosci 2018;12.

21 Milev RV, Giacobbe P, Kennedy SH, et al. CANMAT depression work group. Canadian network for mood and anxiety treatments (CANMAT) 2016 clinical guidelines for the management of adults with major depressive disorder: section 4. neurostimulation treatments. Can J Psychiatry 2016;61:561-75.

22 Lefaucheur JP, Antal A, Ayache SS, et al. Evidence-Based guidelines on the therapeutic use of transcranial direct current stimulation (tDCS). Clin Neurophysiol 2017;128:56-92.

23 Oakes P, Loukas M, Oskouian RJ, et al. The neuroanatomy of depression: a review. Clin. Anat. 2017;30:44-9.

24 Brunoni AR, Ferrucci R, Fregni F, et al. Transcranial direct current stimulation for the treatment of major depressive disorder: a summary of preclinical, clinical and translational findings. Prog Neuropsychopharmacol Biol Psychiatry 2012;39:9-16.

25 Brunoni AR, Moffa AH, Fregni F, et al. Transcranial direct current stimulation for acute major depressive episodes: meta-analysis of individual patient data. Br J Psychiatry 2016;208:522-31.

26 Kalu UG, Sexton CE, Loo CK, et al. Transcranial direct current stimulation in the treatment of major depression: a meta-analysis. Psychol Med 2012;42:1791-800.

27 Berlim MT, Van den Eynde F, Daskalakis ZJ. Clinical utility of transcranial direct current stimulation (tDCS) for treating major depression: a systematic review and meta-analysis of randomized, double-blind and sham-controlled trials. J Psychiatr Res 2013;47:1-7.

28 Meron D, Hedger N, Garner M, et al. Transcranial direct current stimulation (tDCS) in the treatment of depression: systematic review and meta-analysis of efficacy and tolerability. Neurosci Biobehav Rev 2015;57:46-62.

29 Shiozawa P, Fregni F, Benseñor IM, et al. Transcranial direct current stimulation for major depression: an updated systematic review and meta-analysis. Int. J. Neuropsychopharm. 2014;17:1443-52. Erratum in: Int J Neuropsychopharmacol 2014;17(9):1539.

30 Blumberger DM, Tran LC, Fitzgerald PB, et al. A randomized doubleblind sham-controlled study of transcranial direct current stimulation for treatment-resistant major depression. Front Psychiatry 2012;3.

31 Palm U, Schiller C, Fintescu Z, et al. Transcranial direct current stimulation in treatment resistant depression: a randomized doubleblind, placebo-controlled study. Brain Stimul 2012;5:242-51.

32 Bennabi D, Nicolier M, Monnin J, et al. Pilot study of feasibility of the effect of treatment with tDCS in patients suffering from treatmentresistant depression treated with escitalopram. Clin Neurophysiol 2015;126:1185-9.

33 Ferrucci R, Bortolomasi M, Vergari M, et al. Transcranial direct current stimulation in severe, drug-resistant major depression. $J$ Affect Disord 2009;118:215-9.

34 Palm U, Hasan A, Strube W, et al. tDCS for the treatment of depression: a comprehensive review. Eur Arch Psychiatry Clin Neurosci 2016;266:681-94.

35 Boggio PS, Rigonatti SP, Ribeiro RB, et al. A randomized, doubleblind clinical trial on the efficacy of cortical direct current stimulation for the treatment of major depression. Int $J$ Neuropsychopharmacol 2008;11:249-54.

36 Brunoni AR, Sampaio-Junior B, Moffa AH, et al. The escitalopram versus electric current therapy for treating depression clinical study (ELECT-TDCS): rationale and study design of a non-inferiority, triple-arm, placebo-controlled clinical trial. Sao Paulo Med J 2015;133:252-63.

37 Loo CK, Alonzo A, Martin D, et al. Transcranial direct current stimulation for depression: 3-week, randomised, sham-controlled trial. Br J Psychiatry 2012;200:52-9.

38 Dondé C, Amad A, Nieto l, et al. Transcranial direct-current stimulation (tDCS) for bipolar depression: a systematic review 
and meta-analysis. Prog Neuropsychopharmacol Biol Psychiatry 2017;78:123-31.

39 D'Urso G, Dell'Osso B, Rossi R, et al. Clinical predictors of acute response to transcranial direct current stimulation (tDCS) in major depression. J Affect Disord 2017;219:25-30.

40 Tran BX, Ha GH, Vu GT, et al. Indices of change, expectations, and popularity of biological treatments for major depressive disorder between 1988 and 2017: a Scientometric analysis. Int J Environ Res Public Health 2019;16:2255.

41 Dell'Osso B, Dobrea C, Arici C, et al. Augmentative transcrania direct current stimulation (tDCS) in poor Responder depressed patients: a follow-up study. CNS Spectr 2014;19:347-54.

42 American Psychiatric Association. Dsm-5: diagnostic and statistical manual of mental disorders. 5th ed. Washington, DC: American Psychiatric Association, 2013.

43 Roussel C, Carbonneil C, Audry A. Participants of Giens XXXI, round table No. 4. organisational impact: definition and assessment methods for medical devices. Therapie 2016;71:69-96.

44 Manca A, Hawkins N, Sculpher MJ. Estimating mean QALYs in trialbased cost-effectiveness analysis: the importance of controlling for baseline utility. Health Econ 2005;14:487-96.

45 Haute Autorité de Santé. Choices in methods for economic evaluation. Saint Denis La Plaine, France, 2012. Available: https:// www.has-sante.fr/upload/docs/application/pdf/2012-10/choices_in_ methods_for_economic_evaluation.pdf

46 Glick HA. Sample size and power for cost-effectiveness analysis (Part 1). Pharmacoeconomics 2011;29:189-98.

47 Brunoni AR, Ferrucci R, Bortolomasi M, et al. Interactions between transcranial direct current stimulation (tDCS) and pharmacological interventions in the major depressive episode: findings from a naturalistic study. Eur Psychiatry 2013;28:356-61.

48 Faria R, Gomes M, Epstein D, et al. A guide to handling missing data in cost-effectiveness analysis conducted within randomised controlled trials. Pharmacoeconomics 2014;32:1157-70.

49 Gray A, Clarke P, Wolstenholme J, et al. Applied methods of costeffectiveness analysis in health care. New York: Oxford University Press, 2011
50 Nguyen K-H, Gordon LG. Cost-Effectiveness of repetitive transcranial magnetic stimulation versus antidepressant therapy for treatment-resistant depression. Value in Health 2015;18:597-604.

51 Voigt J, Carpenter L, Leuchter A. Cost effectiveness analysis comparing repetitive transcranial magnetic stimulation to antidepressant medications after a first treatment failure for major depressive disorder in newly diagnosed patients - A lifetime analysis. PLoS One 2017;12:e0186950.

52 Ghiasvand H, Moradi-Joo M, Abolhassani N, et al. Economic evaluation of resistant major depressive disorder treatment in Iranian population: a comparison between repetitive transcranial magnetic stimulation with electroconvulsive. Med J Islam Repub Iran 2016;30.

53 Zhao YJ, Tor PC, Khoo AL, et al. Cost-Effectiveness modeling of repetitive transcranial magnetic stimulation compared to electroconvulsive therapy for treatment-resistant depression in Singapore. Neuromodulation 2018;21:376-82.

54 Etcheverrigaray F, Bulteau S, Machon LO, et al. Hospital production cost of repetitive transcranial magnetic stimulation (rTMS) in the treatment of depression. Rev Epidemiol Sante Publique 2015;63:268-74.

55 Sauvaget A, Tostivint A, Etcheverrigaray F, et al. Hospital production cost of transcranial direct current stimulation (tDCS) in the treatment of depression. Neurophysiol Clin 2019;49:11-18.

56 McLoughlin D, Mogg A, Eranti S, et al. The clinical effectiveness and cost of repetitive transcranial magnetic stimulation versus electroconvulsive therapy in severe depression: a multicentre pragmatic randomised controlled trial and economic analysis. Health Technol Assess 2007;11:1-54.

57 Martin DM, Alonzo A, Ho K-A, et al. Continuation transcranial direct current stimulation for the prevention of relapse in major depression. $J$ Affect Disord 2013;144:274-8

58 Valiengo L, Benseñor IM, Goulart AC, et al. The sertraline versus electrical current therapy for treating depression clinical study (select-TDCS): results of the crossover and follow-up phases. Depress Anxiety 2013;30:646-53.

59 D'Urso G, Mantovani A, Micillo M, et al. Transcranial direct current stimulation and cognitive-behavioral therapy: evidence of a synergistic effect in treatment-resistant depression. Brain Stimul 2013;6:465-7. 